\title{
El sudeste asiático: estructura y cambio de sus relaciones internacionales
}

\author{
. \\ Pui See Tang \\ Manfred Wilhelmy von Wolff \\ Lina María Fajardo Vallejo
}

Resumen

El ensayo presenta una visión general de las relaciones internacionales del sudeste asiático, con el fin de identificar las principales características de su vinculación con el este de Asia y las principales potencias de la región. La primera parte del texto explora los orígcnes del término «sudeste asiático" y del orden regional anterior a la Segunda Guerra Mundial. La segunda y tercera partes se centran en los desarrollos de la guerra y de la post Guerra Fría, haciendo hincapié en el rol de la Asociación de Naciones del Sudeste Asiático (ASEAN) como medio a través del cual sus miembros se insertan en la región del Asia Pacífico. La cuarta sección explora las relaciones del sudeste asiático con las principales potencias: Estados Unidos, China y Japón.

Abstract

The essay addresses the international relations of the Southeast Asian region, with the purpose of identifying the main characteristics of its interaction with East Asia and the regional powers. The first part explores the origins of the term "Southeast Asia" and the regional order pre- World War II. The second and third sections focus on Cold War and post-Cold War developments, stressing the role of the Association of Southeast Asian Nations (ASEAN) as a vehicle for Southeast Asian relations with the Asia Pacific region. The fourth section examines Southeast Asian relations with the main powers: United States, China and Japan. 


\section{INTRODUCCIÓN}

Este ensayo presenta una visión general de las relaciones internacionales del sudeste asiático, subregión integrada por los países miembros, la Asociación de Estados del Sudeste Asiático (ASEAN). Específicamente, se trata de determinar las características centrales de la interacción de los países que conforman esta subregión y su articulación en la dinámica del este asiático. Esta se ha transformado profundamente con el surgimiento de China como actor de primer orden, junto al protagonismo de Estados Unidos en la post Guerra Fría y la búsqueda de un rol internacional más asertivo por parte de Japón.

En la primera parte del ensayo se reseña el origen del tếrmino "sudeste asiático» y el orden regional en los años anteriores a la Guerra Fría. Se argumenta que el sudeste asiático como concepto es relativamente nuevo, no así el orden regional que se construye a través de la interacción de los reinos, las potencias coloniales y el proceso de descolonización. La segunda y tercera parte se centran en los años de la Guerra y post Guerra Fría, poniendo énfasis en el rol de ASEAN como medio a través del cual la región adquiere identidad propia y se proyecta y articula en la dinámica del este asiático. Cabe destacar que la ASEAN ha sido fundamental en la creación de normas de socialización y en la construcción de una comunidad, constituyendo $y$ transformando el orden regional en el sudeste asiático. La cuarta sección se detiene en las relacio- nes del sudeste asiático con las principales potencias: Estados Unidos, China y Japón. En este contexto, se argumenta que el sudeste asiático emplea una doble estrategia de maximizar los beneficios económicos de la presencia de las potencias en la región, al mismo tiempo que se minimizan sus posibles efectos negativos.

\section{ORIGEN DEL TÉRMINO «SUDESTE ASIÁTICO»}

El término «sudeste asiático» (en adelante SEA), es de reciente origen. La subregión fue concebida durante la Segunda Guerra Mundial en términos geopolíticos y militares por los aliados, que enfrentaban el desafío de vencer a los japoneses que se habian apoderado del área. Entre 1941 y 1944, la National Geographic Society distribuyó millones de copias de mapas del «sudeste asiátjco", término acuñado combinando las nociones de «sur» $y$ «este» del Asia, tanto en el continente como en los archipiélagos colindantes.

Los requerimientos operacionales militares generalizaron la aproximación al área como una región, o subregión, que antes se había visto indistintamente como parte del sur o del este de Asia. De esta manera, el SEA comenzó a adquirir connotación política y surgió como un escenario de acción diplomática y objeto de atención aca démica, comprendiendo -como categoría residuallos países y territorios asiáticos situados al este de la India y al sur de China. 
Cabe destacar que en el SEA precolonial, el orden político internacional no fue algo desconocido aunque no correspondió al modelo europeo de colectividades estatales claramente delimitadas (alllamado "sistema de Westfalia»), sino a un conjunto sociopolítico más flexible y menos estructurado: el sistema de «mandala». Este consistía en una serie de relaciones organizadas en forma de círculos concéntricos - generalmente tres - en los que el rey se ubicaba al centro, sin ejercer poderes absolutos y sin un área de dominación territorial muy consolidada. La mandala evolucionó como una serie continua de reinos, a veces imperios, cuyas esferas de influencia se superponían en diversos grados. La tónica ideal de comportamiento en este sistema era evitar los conflictos y desarrollar consensos, aunque, obviamente, en la práctica las cambiantes relaciones de poder dieron lugar a numerosas confrontaciones diplomáticas y situaciones bélicas, especialmente condicionadas por el control real de los monarcas (primer círculo), príncipes, otros señores y vasallos (segundo círculo) sobre las zonas periféricas de sus reinos (tercer círculo de la mandala). En este sistema se ha visto la raíz de lo que se ha denominado "el estilo ASEAN", que enfatiza la construcción de consensos y el control de los conflictos.

El colonialismo alteró de manera fundamental el orden regional, en el sentido de que la interdependencia con el mundo externo pasó a predominar sobre los antiguos lazos regionales. Las redes comerciales asiáticas tendieron a desintegrarse; el SEA se fragmentó al desarrollarse una red de comunicaciones de larga distancia que obedecía a imperativos coloniales. Las fronteras establecidas por las potencias coloniales reflejaron las relaciones de poder entre estas y dieron origen a sociedades étnica y culturalmente mixtas, $y^{\prime}$ a la división de grupos existentes entre diferentes colonias. De esta manera, la «edad del comercio» precolonial de los siglos XV a XVII (Anthony Reid) tocó a su fin. Especialmente a partir de las primeras décadas del siglo XIX, las fronteras coloniales exclusivas con cada metrópoli sirvieron los intereses respectivos, así como el "equilibrio europeo". Las diferencias locales históricas entre diferentes segmentos del SEA, marcados por influencias indias, chinas y filipinas perdieron importancia. Este proceso, a su vez, nutrió el desarrollo posterior de un sentimiento nacionalista común, expresado en aspiraciones de fundar Estados independientes e impulsar proyectos de modernización.

La conquista japonesa del SEA a partir de 1942 estimuló la conciencia regional, tanto porque Tokio promovió el antioccidentalismo como porque por primera vez el SEA se encontró bajo un solo gobierno, parte de la «esfera de coprosperidad" regida por Japón. ${ }^{3}$ Por su parte, los aliados crearon el «Southeast Asian Command» (SEAC), nomen-

! Así se puso fin a la "partición" colonial. 
clatura que contribuyó a difundir el término «SEA» en los Estados Unidos y otros países. Entre los académicos, el tema del SEA pasó a ser parte de los estudios regionales políticos, económicos y culturales en las universidades y centros de investigación.

El fin de la Segunda Guerra Mundial sirvió de catalizador en la búsqueda de la independencia de los poderes regionales y la creación de identidades nacionales. La descolonización constituyó un movimiento antieuropeo en los planos nacional y regional. En 1947 se celebró en Nueva Delhi una Conferencia de Relaciones Asiáticas, en la que participaron activamente delegaciones del SEA. No obstante, el potencial de un movimiento panasiático se vio afectado por las divisiones entre percepciones y elementos procomunistas, prooccidentales y neutralistas, mientras que a nivel nacional los movimientos insurgentes comunistas, combinados con diversas manifestaciones separatistas, estimularon reacciones nacionalistas conservadoras y una tendencia regional a la unificación de acuerdo con modelos políticos autoritarios. Asimismo, la emancipación de las colonias del área estimuló la intervención de las nuevas autoridades políticas en los sistemas económicos pertinentes.

Los imperativos de estabilidad y seguridad se impusieron bajo diferentes fórmulas políticas más o menos alejadas de los modelos democráticos, en Malasia, Indonesia, Singapur y Filipinas. Tailandia -que no conoció la experiencia colonial-vivió frecuentes epi- sodios de inestabilidad e intervención militar en sus procesos políticos. Gran Bretaña abogó ante Estados Unidos por una iniciativa regional equivalente a lo que había sido el Plan Marshall en Europa, propuesta que no tuvo acogida en Washington.

\section{Guerra Fría y regionalismo}

En los primeros años de la postguerra, el SEA no fue prioridad para Estados Unidos ni para la Unión Soviética. En el Reino Unido circularon las primeras versiones de lo que más adelante se conocería como «teoría del dominó» -la instauración del régimen comunista en China podría llegar a provocar la caída de Indochina, Birmania, Tailandia y Malasia. En su momento, Washington incluyó al SEA en su "política de contención" anticomunista, y estableció la organizaciốn del Tratado del Asia Sudoriental (SEATO, por su sigla en inglês), especie de adaptación regional del modelo OTAN. Esta entidad no alcanzó la prominencia de este ente defensivo, ya que nunca llegó a contar con una base política regional sólida.

En los años sesenta, el anuncio de la retirada militar británica de los territorios situados al oriente del canal de Suez, la "doctrina Nixon", que buscaba desvincular a Estados Unidos de compromisos estratégico-militares abiertos e indefinidos en el SEA, la creciente división chino-soviética y el temor al surgimiento del poder chino, estimularon el desarrollo de un regio- 
nalismo centrado en los recursos políticos y económicos de los países del área, que compartían la preocupación por el peligro que representaban diversos movimientos insurgentes a lo largo de sus fronteras.

Al interior del SEA, la caída del gobierno de Sukarno en Indonesia, en 1967, eliminó el peligro de la «Konfrontasi» ${ }^{2}$, y el nuevo régimen de Jakarta (del general Suharto) consideró que la cooperación regional era indispensable para sus planes de desarrollo, así como para proyectar influencia en el SEA. Por su parte, Singapur vio en la cooperación regional la posibilidad de legitimarse como Estado y controlar su alto grado de vulnerabilidad internacional, consecuencia de su reducido tamaño y de su fórmula política interna. Esta se basaba en un pacto de gobierno multiracial entre la mayoría china y las minorías malayas e indias, en contraste con la hegemonía malaya de los países vecinos. Tailandia, Malasia y Filipinas consideraron que la cooperación regional podría contribuir a la pacificación de la zona y a desarrollar relaciones basadas en un mejor nivel de confianza recíproca.

Esta convergencia de motivaciones e intereses llevó a que el 9 de agosto de 1967, Tailandia, Malasia, Indonesia, Singapur y Filipinas aprobaran la $\mathrm{De}$ claración de Bangkok, en virtud de la cual se estableció la ASEAN. Birmania (hoy Myanmar) y Camboya rehusaron participar por estimar que la ASEAN era un bloque prooccidental y eventualmente anti-chino.

De acuerdo con la Declaración de Bangkok, la ASEAN era una «solución regional a problemas regionales». Sus objetivos formales fueron la aceleración del crecimiento económico, el progreso social y el desarrollo cultural, junto con la promoción de la paz y la estabilización regionales. En la práctica, estos últimos objetivos han sido prioritarios para la organización, ya que durante mucho tiempo la cooperación fue una aspiración programática.

En la Declaración, los Estados de la ASEAN manifestaron su voluntad de asegurar su estabilidad y seguridad $\sin$ intervenciones externas. Sin embargo, al momento de ser aprobada todos sus miembros, salvo Indonesia, tenían bases militares extranjeras en su territorio. En este contexto, en 1971, la ASEAN promulgó la Declaración sobre la Zona de Paz, Libertad y Neutralidad (ZOPFAN), que constituye una visión o posición de principios, en la que se afirma que el SEA debe lograr gradualmente el control del destino de la región. Indonesia influyó para que se expresara el principio de que todas las bases extranjeras serían transitorias y solo podían permanecer con la expresa voluntad de cada país.

La ZOPFAN expresó una postura política alternativa frente a la división del SEA en una zona pro-occidental,

2 La «Konfrontasi» fue una confrontación por la propiedad de la isla de Borneo entre Inclonesia y Malasia, entre 1962 y 1966. La gucrra estalló en 1963 y se prolongó hasta agosto de 1966, cuando ambos países firmaron un tratado de paz. 
bajo la influencia de las políticas de Estados Unidos, y una zona (Indochina) expuesta a la actividad soviética y china. No obstante, el conflicto de Vietnam, y en general de Indochina, relegó esta idea al plano teórico, especialmente a partir de 1978, cuando la invasión vietnamita en Camboya polarizó fuertemente al SEA.

En la Cumbre de Bali, de 1976, la ASEAN aprobó el Tratado de Amistad $y$ Cooperación, que constituyó un pacto de no agresión entre sus miembros y formalizó un código de conducta entre ellos, que incluyó los principios básicos de respeto mutuo por la independencia, la soberanía y la integridad territorial de los miembros; el derecho de cada uno a conducir sus asuntos sin estar sujeto a la coerción de otro ni verse afectado por situaciones subversivas instigadas desde el exterior; la no intervención en los asuntos internos, la solución pacífica de las controversias y la renuncia a la amenaza de uso de la fuerza en las relaciones reciprocas ${ }^{3}$.

En este contexto, ASEAN buscaba equilibrar la visión de una subregión responsable de su propio destino con el mantenimiento de un apoyo tácito al rol de los Estados Unidos. No cabe duda que, tal como sigue ocurriendo hoy, algunos miembros de la ASEAN preferían contar con una presencia significativa de Estados Unidos. Por ejemplo, el fundador del Estado de Singapur, Lee
Kuan Yew, sostenía que dada la situación geopolítica de la zona, la presencia estadounidense en algunas bases navales y aéreas podía ayudar a mantener cierto equilibrio. Esta consideración, así como motivaciones comerciales, explican la decisión de Singapur, a comienzos de los años noventa, de prestar servicios de recalada a la Séptima Flota de la Armada de Estados Unidos cuando esta debió cerrar la base naval de la bahía de Subic, en Filipinas, a solicitud del gobierno de ese país.

Cabe destacar que en el SEA el tema de la seguridad ha sido una constante en las actuaciones de la ASEAN desde sus inicios. La seguridad se concibe en términos amplios, incluye aspectos políticos, militares, económicos y sociales y sirve para mitigar las tensiones entre sus miembros; eliminar a través del desarrollo económico las condiciones sociales que generan los movimientos insurgentes; y reducir la vulnerabilidad de sus miembros a las políticas de las grandes potencias ${ }^{4}$. Igualmente, la ASEAN ha desarrollado lo que se ha conocido como 'estilo ASEAN', un tipo de interacción basado en la toma de decisiones por consenso, que ha funcionado también como método de resolución de conflictos al interior del organismo y le ha permitido mantener su unidad $^{5}$.

Hacia el fin de la Guerra Fría, la ASEAN había logrado articular una

3 Naurine, S. (1998), "ASEAN and the management of regional security", Pacific Affairs, $71(2)$, p. 201.

4 Ibid., p. 196.

s Ibid., p. 202. 
identidad regional. El esfuerzo consciente de los líderes de la región de construir e imaginar un orden regional es una de las características centrales de esa identidad, que incluye también el evitar los conflictos y la resolución pacífica de estos ${ }^{6}$. En la crisis de Camboya, esto le dio una voz importante al organismo, que con sus actuaciones diplomáticas contribuyó a contener la política vietnamita de expansión en ese país, lo cual era percibido como una amenaza importante, especialmente en Tailandia. Cuando más adelante Hanoi perdió su aliado soviético, ya no contó con suficiente apoyo material y diplomático para esta aventura y debió retirar sus fuerzas militares de Camboya. El episodio contribuyó a la maduración de la ASEAN y a que la entidad fuera considerada como el experimento regionalista más exitoso del Tercer Mundo, capaz de intervenir para mantener la seguridad en su entorno.

\section{La post Guerra Fría: paz Y PROSPERIDAD}

La ASEAN procura alcanzar una relación positiva entre el desarrollo económico y la seguridad regional. La solución del conflicto camboyano permitió que ese país, junto con Laos y Vietnam, se integraran a la institucionalidad de la región. La paz favoreció el desarrollo económico del SEA y multiplicó las vinculaciones con otras regiones del este de Asia. Al interior de la ASEAN, a partir de los años noventa tomaron impulso las zonas transnacionales (transfronterizas) de crecimiento económico (como el «triángulo de crecimiento" Singapur-Johor, Malasia-Batam, Indonesia), que son experiencias de integración regional novedosas y pragmáticas.

Sin embargo, han persistido factores de incertidumbre. A raíz de la salida de Estados Unidos de sus bases militares en Filipinas, en 1991, su papel como proveedor de la seguridad en el SEA no esté garantizado, mientras que un hipotético crecimiento de la presencia militar nipona en la zona enfrenta fuerte resistencia debido a la general desconfianza producto de las experiencias del pasado. El enorme crecimiento económico de China agrega nuevas interrogantes a la problemática regional, ya que la retirada de la ex Unión Soviética del este de Asia y al perfil más bajo de Estados Unidos en la región, $\sin$ duda han favorecido la posición geopolítica de ese país.

La estrategia de la ASEAN para enfrentar dichos factores ha contemplado cuatro elementos. En primer lugar, amplió la posibilidad de participar en el organismo a los países de la subregión que militaban en el campo socialista: Vietnam en 1995, Laos y Myanmar en 1997 y Camboya en $1999^{7}$. De esta manera, culminó el sueño de sus fun-

6 Acharya, A., «Do norms and identity matter? Community and power in Southeast Asia's regional order", The Pacific Review, 18 (1), p. 104.

7 Brunei Darussalam ingresó a ASEAN en 1984. 
dadores de agrupar a todos los países del SEA ${ }^{8}$. La existencia de 'un' SEA, es un verdadero hito en la historia de la región pues, como se ha visto, durante la ocupación japonesa la región solo estuvo integrada brevemente, si bien bajo la dominación extranjera, lo cual lo diferencia de esta nueva situación ${ }^{9}$.

En segundo lugar, ha profundizado la cooperación económica mediante el proceso de reducción de los aranceles a partir de la suscripción del acuerdo sobre la creación del Área de Libre Comercio de ASEAN (AFTA) en 1992. Sin embargo, este proceso de liberalización no ha sido básicamente amplio y en general se ha limitado principalmente a productos manufacturados. Las metas son alcanzar el arancel cero para Indonesia, Malasia, Singapur; Tailan-dia, Filipinas y Brunei el año 2010 y el 2015 para Vietnam y el resto de la ASEAN. Lo anterior se complementa con el compromiso de permitir ciertos niveles de protección a algunos productos considerados «sensibles» hasta el año 2018.

En su reunión del 2006, los ministros de Comercio acordaron adelantar la fecha de vigencia del mercado único de la ASEAN (sin moneda regional) del año 2020 al 2015. Esta decisión revela la preocupación de los gobiernos por un eventual rezago de la ASEAN frente a las principales potencias comerciales, así como el deseo de no perder terreno en el contexto de los desafíos económicos que plantean China e India.

En tercer lugar, el campo de la economía y de la seguridad, la ASEAN ha aplicado deliberadamente una política de promoción del regionalismo en el este de Asia y ha procurado fortalecer sus relaciones externas a través de instancias de diálogo con Estados Unidos, Australia, Japón, China, Corea del Sur y la Unión Europea. De esta manera, se ha posicionado como motor del regionalismo en el este de Asia y ha impuesto su estilo en todas estas agrupaciones. En el campo económico, cabe destacar la creación de ASEAN + tres, mecanismo de diálogo de sus miembros con Japón, China y Corea; la creación de la Cumbre del Este de Asia que incluye además a India, Australia, Nueva Zelandia; y el establecimiento de la ASEAN como núcleo central del APEC, motivo por el cual año por medio uno de sus miembros debe presidirlo.

En el campo de la seguridad creó el Foro Regional de la ASEAN (ARF), instancia de consulta y diálogo diplomático de los países del SEA con los principales actores internacionales en esta materia. El programa del ARF contempla tres fases: construcción de confianza, diplomacia preventiva y solución de conflictos. Adicionalmente, promueve la adopción de un Tratado de Amistad y Cooperación como código

8 Chia, Siow Yue; Soesastro, H. (2006), "ASEAN perspectives on promoting regional and global freer trade", documento inédito, p. 134.

9 Djalal, H. (1997), "One Southeast Asia in World Affairs", en Soesastro, H. (ed.), One Southeast Asia in a new regional and international setting, Jakarta, Centre for Strategic and International Studies, p. 154. 
de conducta para las relaciones interestatales ${ }^{10}$.

Es importante recalcar que la cooperación y la integración regionales en el este de Asia se conciben como instrumentos para lograr la paz, prosperidad $y$ equidad en la zona, puesto que fomentan la acción conjunta en asuntos tales como el impulso al crecimiento económico, la reducción de la pobreza y la eliminación de la disparidad en los niveles de desarrollo." ${ }^{11}$

Finalmente, en 1997 la ASEAN promulgó la Visión 2020, mediante la cual se propone crear un concierto de naciones del SEA, orientado al exterior, que convivan en paz, estabilidad y prosperidad, unido mediante el desarrollo económico común y el sentimiento de comunidad. Para desarrollar esta visión, en 2003 la ASEAN se comprometió mediante el Acuerdo de Bali II a crear una Comunidad de la ASEAN para el año 2020. Esta se basaría en tres ejes: económico, seguridad y sociocultural ${ }^{12}$. La ASEAN ha entendido la comunidad económica como la manera de competir $y$ proyectarse como una alternativa a la emergencia de China e India como po- tencias económicas ${ }^{13}$. La comunidad en materia de seguridad apunta a construir sobre las experiencias del pasado y asegurar que la convivencia armoniosa de los países del SEA y que no se recurra al uso de la fuerza para resolver disputas. El eje sociocultural tiene por finalidad el mejorar las condiciones de vida de los pueblos del SEA, mediante el disfrute de los beneficios de la integración económica ${ }^{14}$.

\section{LAS RELACIONES DEL SEA CON LAS POTENCIAS}

Los miembros de la ASEAN están conscientes de que la presencia de China, Estados Unidos y Japón en su región es una realidad, por lo cual aplican una estrategia consistente en maximizar los beneficios económicos de su relación con las potencias, al tiempo que minimizan los riesgos en materia de seguridad al evitar que alguna de ellas se constituya en hegemónica en la región. Mediante ese delicado acto de equilibrio, la ASEAN procura alcanzar sus metas de desarrollo económico, paz y prosperidad. ${ }^{15}$

10 Naurine, S. (1998), p. 209.

1 Kuroda, H. (2006), "Toward an Integrated, poverty-frec, and Peaceful East Asia", en Idermit S. Gill, Yukon, Huang, y Homi Kharas (eds.), East Asian Visions: Perspectives on Economic Development, Singapur, World Bank and Institute of Policy Studies.

12 ASEAN (2006), Overwiew: Association of Southeast Asian Nations <http:/wwwaseansec.org7147.htm>.

13 Pangestu, M. (2006), "Visions of East Asia: Three Engines the Way Forward", en Idermit S. Gill, Yukon, Huang, y Homi Kharas (eds). op. cit., p. 166.

14 ASEAN (2006).

is Chung, C.P. (2004), "Southeast Asia-China relations: dialectics of 'hedging' and 'counterhedging'", en Southeast Asian Affairs 2004, Singapur, Institute of Southeast Asian Studies, ISEAS, p. 35 . 
Relaciones del SEA con los Estados Unidos

Históricamente, el SEA no ha sido una zona de interés especial para los Estados Unidos. Washington comenzó a prestarle más atención debido a que vio en el SEA a un grupo de países prooccidentales, cuyas políticas económicas eran favorables para sus intereses. La excepción fue Indonesia durante la era de Sukarno. Pero el régimen populista y nacionalista de izquierda del carismático líder indonesio se desmoronó a partir de los sangrientos sucesos de 1965 que llevaron al poder al general Suharto, quien instaló el «Nuevo Orden». En cambio, las alianzas entre Filipinas, Tailandia, Singapur y Estados Unidos se han mantenido estables en el tiempo, con excepción del problema de las bases militares en Filipinas.

En esos países, y en la Indonesia post Sukarno, ha predominado la percepción de Estados Unidos como factor de estabilidad regional. Bajo Suharto la relación bilateral con Jakarta fue muy importante bajo Suharto, pero se vio afectada por problemas en materia de derechos humanos. En Malasia, el gobierno y los principales actores políticos han combinado una retórica de independencia, de críticas a Estados Unidos y de promoción del "pan-asianismo", con el reconocimiento pragmático de la primacía geopolítica de Washington. En la era post-Mahathir (desde fines de 2003), el gobierno del Pri-

16 Chung, C.P. (2004), p. 36.

17 Ibid.y p. 42. mer Ministro Badawi ha dado un giro moderado y pragmático a la política exterior de Kuala Lumpur.

Desde la perspectiva de la ASEAN, Estados Unidos aparece como la superpotencia que subordina sus políticas hacia la subregión a esquemas geopolíticos globales y regionales. En el SEA se percibe claramente que uno de los intereses primordiales de Estados Unidos en la geopolítica del este de Asia es mantener la cooperación estratégica con Japón. Sin discutir el tema, los actores del SEA procuran separar su apoyo a Estados Unidos de un acercamiento a Tokio en el ámbito de la seguridad, ya que persiste la desconfianza y el recelo originados en el comportamiento japonés en la era del imperialismo y el militarismo, y surgen temores de que el fortalecimiento de dicha cooperación pueda generar reacciones chinas que desemboquen en una carrera armamentista ${ }^{16}$. Para los países del SEA la presencia de Estados Unidos en la zona es deseable en la medida en que funcione como resguardo en caso de un comportamiento chino más agresivo en la región ${ }^{17}$.

Al mismo tiempo, se percibe una preferencia de Estados Unidos por las relaciones bilaterales ( $y$ las acciones unilaterales). En este contexto no ha pasado inadvertida, la antigua oposición de Washington a los instrumentos políticos básicos de la ASEAN, como el Tratado de Paz y Amistad (1976), que conlleva una adhesión al concepto de 
la "ZOPFAN" (Zona de Paz, Libertad y Neutralidad). Este concepto no conviene a la postura estratégica global de Estados Unidos, en la medida en que podría implicar un compromiso de debilitar o excluir los entendimientos militares subregionales o bilaterales de Washington en el SEA.

En lo económico, ha aumentado la importancia de la ASEAN para Estados Unidos. En 2002 el comercio con ese país alcanzó a 120.000 millones de dólares. Las exportaciones estadounidenses al área ASEAN duplican con creces las ventas a China y la zona es el quinto mercado de destino de exportaciones de Estados Unidos. La inversión extranjera directa de Estados Unidos en el área ascendió a 50.000 millones de dólares (2002), quintuplicando el valor de la inversión dirigida a China. De acuerdo con cifras de julio de 2006, los países de la ASEAN son el cuarto socio comercial de los Estados Unidos. ${ }^{18}$

En el año 2002 Washington propuso la "Enterprise for ASEAN Initiative" y el «Plan de Cooperación ASEAN», con el fin de establecer un entorno regional de seguridad para apoyar el comercio y la inversión y aumentar la apertura de los mercados de la ASEAN a la oferta exportadora de Estados Unidos. Tambiến apunta a estimular el crecimiento económico del área mediante el fortalecimiento de la integración regional y el apoyo a las reformas económi- cas, financieras, regulatorias y judiciales en su ámbito. Igualmente, Estados Unidos busca institucionalizar su relación con la ASEAN mediante un futuro acuerdo comercial con el organismo, la designación de un embajador en su seno y un plan quinquenal, acordado en julio del 2006, con el fin de fortalecer los lazos políticos y económicos ${ }^{19}$. Todo esto revela que tanto el gobierno como el Congreso de los Estados Unidos tienen ahora más conciencia del rol de la ASEAN y procuran aumentar su presencia en el área.

A nivel bilateral, esta tendencia se ha manifestado en el TLC Estados Unidos-Singapur (vigente), las negociaciones de libre comercio con Tailandia, la firma de un Acuerdo de Facilitación del Comercio y las Inversiones (TIFA) con Malasia, el establecimiento de relaciones comerciales normales con Laos, la incorporación de Camboya a la OMC, $y$ las reciente en el proceso de admisión de Vietnam.

Por último, no cabe duda que los sucesos del 11 de septiembre de 2001 contribuyeron a aumentar la relevancia del SEA para Estados Unidos, tanto porque la subregión es una zona de cierto riesgo terrorista, como porque la ASEAN es un valioso aliado en la Iucha antiterrorista. En este contexto, el 1 de agosto de 2002, la ASEAN y Estados Unidos firmaron una Declaración Conjunta sobre Cooperación Antiterrorista.

18 Simon, S. (2006), "US-Southeast Asian Relations: US strengthens ties to Southeast Asian Nationalism", Comparative Connections: A quarterly E-joumal on East Asian Bilateral Relations, 8 (3), julio-septiembre, p. 64. 
El prisma de la atención de Estados Unidos al SEA destaca las perspectivas de la guerra antiterrorista $y$-secundariamente- los derechos humanos. La política de Estados Unidos es de equilibrio y de conservación del statu quo favorable a sus intereses. Es la línea de comportamiento de una potencia hegemónica que percibe el crecimiento del poder relativo de otros grandes actores, pero que se opone al surgimiento de otro proyecto potencialmente hegemónico.

El énfasis de Estados Unidos en las políticas y alianzas antiterroristas ha complicado las relaciones con la región en la medida en que varios de los países fundadores de la ASEAN tienen importantes poblaciones musulmanas y por tanto se asocian con mayores riesgos en el nuevo contexto político-estratégico (Indonesia, Malasia y Filipinas especialmente). Este enfoque restrictivo de los Estados Unidos en las operaciones antiterroristas desconoce la existencia de un amplio sector social y político musulmán moderado, que responde a una corriente más general del mundo islámico. A este segmento le preocupan, por ejemplo, disparidades socioeconómicas que no parecen tener suficiente reconocimiento en las políticas de los Estados Unidos. Obviamente el ajuste de este enfoque exigiría revisar la estrategia de Washington en otras áreas, especialmente el Medio Oriente.

\section{Relaciones del SEA con China}

Históricamente, China solo percibía la región en términos de la noción de «Indochina». En el SEA predominaba la desconfianza hacia China debido a las reales o presuntas vinculaciones de los partidos comunistas locales con ese país. Las minorías chinas o de origen chino en el SEA («chinos de ultramar») eran impopulares, en gran medida por su riqueza relativa, especialmente en los países predominantemente malayos, $y$ se sospechaba que algunos chinos también podían ser vehículos de influencia de China en la zona.

El interés chino se dirigió en primer término a Tailandia e Indonesia, por consideraciones de seguridad y económicas (acceso a materias primas). Malasia estableció relaciones diplomáticas con China en 1974; Tailandia y Filipinas lo hicieron en 1975. Indonesia demoró este paso hasta agosto de 1990. Singapur, que por razones diplomáticas estimaba que no podía adelantarse a Jakarta, lo hizo dos meses después.

La percepción inicial de China sobre la ASEAN fue la de una entidad hostil, anticomunista y pro-occidental. Sin embargo, a partir de su proceso de reforma y apertura, ha aumentado su interés en la región. Para China, es importante que el SEA no esté alineado con alguna potencia, sea regional o no, que pueda tener políticas hostiles hacia ella. Por lo tanto, su estrategia en la región busca atraer a los países del SEA, disipar sus temores acerca de una China económica y políticamente fuerte, $y$ di- 
ficultar la presencia de Estados Unidos o Japón en la zona. Por su parte, la ASEAN aplica una estrategia de cooperación económica con China, al mismo tiempo que evita compromisos estratégicos con ella, como forma de enfrentar la incertidumbre generada por su reemergencia ${ }^{20}$.

La invitación a China a que participe en eventos vinculados a la Conferencia Ministerial de la ASEAN, formulada en 1991, y la participación china en el Foro Regional de la ASEAN desde 1994, marcaron grandes progresos de la diplomacia de Beijing y fue una muestra de realismo de parte de los gobiernos de la ASEAN. A partir de 1997, la ASEAN y China han celebrado cumbres oficiosas (ya el año anterior China se había convertido en "dialogue partner» oficial de la ASEAN). Las partes han firmado un Tratado de Libre Comercio (que irá materializando gradualmente), y desde 2003 suscriben el concepto de "asociación estratégica para la paz y la prosperidad». Es importante destacar que esa misma China (seguida por Japón e India), adhirió al Tratado de Amistad y Cooperación de la ASEAN y fue el primer país de fuera de la región en ser parte de él. De esta manera, China aceptó que sus relaciones se rigieran por el código de conducta de la ASEAN, lo cual es considerado por el organismo como un gran éxito ${ }^{21}$. Como con- traparte, en el Comunicado conjunto sobre la reunión oficiosa de Ministros de Relaciones Exteriores de la ASEANy China emitido en 2004, la ASEAN reafirmó explícitamente la existencia de una sola China ${ }^{22}$.

China ha dejado atrás su pasado confrontacional en el SEA, que atribuía prioridad a los aspectos militares y territoriales y al ejercicio de influencia a través de los partidos comunistas. Actualmente, a China le interesa sobre todo asegurar su acceso a los recursos naturales del SEA -especialmente energéticos- para continuar su rápida trayectoria económica ascendente. Al convertirse en gran importador de commodities, así como en exportador de manufacturas, China comparte con otras potencias comerciales del este de Asia, incluido el SEA, un interés adicional muy importante: mantener abiertas y seguras las rutas de tránsito marítimo entre China y sus principales contrapartes comerciales.

Aunque en algunos rubros China y la ASEAN son competidores comerciales, el «boom» de la economía china ha estimulado fuertemente el comercio entre ambos. A partir de 1993, el comercio China-ASEAN ha aumentado vertiginosamente. El acuerdo marco ASEAN-China (2002), contempla la desgravación del intercambio, en el período 2005-2015 que podría culminar

20 Chung, C. P. (2004), p. 37-38.

21 Stubbs, R. (2004), "ASEAN in 2003: adversity and response", en Southeast Asian Affairs 2004, Singapur, Institute of Southeast Asian Studies, ISEAS, p. 8.

22 ASEAN (2004), "ASEAN China Foreign Ministers" Informal Meeting Joint Press Release 21 June 2004», Qingdao, China <http://www.aseansec.org/16167.htm>. 
con un área de libre comercio, la mayor del mundo en términos de población.

El énfasis chino en las relaciones económicas y' especialmente en el comercio es parte de una política que procura perfilar a la República Popular China como un actor moderado, políticamente confiable. En el plano territorial, esto conlleva una actitud no confrontacional en el diferendo sobre el archipiélago Spratly, área del Mar del Sur de la China que es reclamada por varios miembros de la ASEAN y por China. Como parte de esta política, en 2002 China y la ASEAN firmaron una Declaración sobre un Código de Conducta para las partes en el Mar del Sur de China, en la que se establecen parámetros de cooperación y exploración conjunta de los recursos naturales de la zona. Aunque la declaración no es vinculante y no resuelve el problema de la soberanía sobre las islas, el hecho de que las partes hayan accedido a firmarla es de por sí, significativo. ${ }^{23}$

Beijing también se ha esforzado en aparecer como un país asiático solidario con sus vecinos. En el marco de la crisis financiera asiática, mientras Estados Unidos insistía en enfrentar la situación mediante programas de ajuste diseñados por el FMI, China ofrecía paquetes de asistencia bilateral. Frente a las medidas de sanción contempladas en diversas leyes de Estados Unidos, que se han aplicado o se ha amenazado con aplicar a la mayoría de los diez Estados miembros de la ASEAN, China ha reaccionado esgrimiendo el argumento de la comunidad de intereses. Por ejemplo, cuando el gobierno militar de Myanmar (Birmania) volvió a arrestar a la líder opositora Aung San Suu Kyi (2003), Washington impuso sanciones más estrictas que las entonces vigentes, mientras que China ofreció asistencia por 200 millones de dólares. China también apoyó exitosamente al régimen birmano frente a los países europeos, que no veían con buenos ojos el ingreso de Myanmar al sistema de diálogo asiático europeo ASEM (Asia-Europe Meetings). Obviamente, aquí ha coincidido el interés chino en aparecer como un importante país amigo asiático, con el deseo, de origen interno, de no sentar precedentes de tipo democratizador o de acción colectiva en favor de los derechos políticos y de un cambio de régimen.

Un elemento básico de la política china frente al SEA ha sido perfilarse como un país vecino que respeta la autonomía de los países de la subregión y de la ASEAN como agrupación, sin buscar relaciones exclusivas o excluyentes. La ASEAN ha visto a China como un actor que no se opone a que se cultiven otras relaciones que equilibran las crecientes vinculaciones con Beijing, y que tampoco busca frenar el crecimiento de la ASEAN. Por ejemplo, China no se opuso al ingreso de Vietnam, su antiguo adversario, a la ASEAN, mientras que durante la Guerra Fría había visto

23 Chung, C.P. (2004), p. 39. 
a Hanoi, aliado de Moscú, como una amenaza. De este modo-dejando atrás la incidencia del factor soviético a través de Vietnam-la ASEAN ganó su propio espacio internacional, aspirando a promover dentro de este sus intereses comunes. China percibió certeramente esta aspiración y procuró reaccionar positivamente frente a ella.

El análisis de la política china revela motivaciones pragmáticas. Ese país, consciente de la percepción de vulnerabilidad que surgió en la ASEAN frente a Beijing, procuró contrarrestar cualquier reacción adversa con su tesis del «surgimiento pacíficon. En términos de dicha tesis, el interés chino radica en la paz y la estabilidad internacionales, ya que esas condiciones son esenciales para el avance de las políticas orientadas a lograr las ambiciosas metas de desarrollo del país. China aspira a que su «surgimiento pacífico" no despierte resistencias en una zona tradicionalmente muy sensible a la proyección del poder chino, en la que además reside una numerosa diáspora (los "chinos de ultramar», que serían de 30 a 40 millones), minoría que es próspera e influyente pero que, como se ha dicho, en varios países del SEA también es vulnerable.

Oficialmente, los gobiernos de la ASEAN han tomado conocimiento de esta noción. No obstante, cada uno de ellos percibe claramente que, a diferencia de lo que ocurría en el pasado, China es una gran potencia de enorme gra- vitación en toda la región, y es tomada en cuenta en todos los aspectos de la diplomacia regional pese a sus promesas de evitar actitudes de tipo "hegemónicon. En privado, algunos actores de la ASEAN suelen expresar dudas sobre el perfil geopolítico de China en el futuro. Vietnam, que en décadas anteriores se beneficiaba con las diferencias entre Beijing y Moscú, ya no dispone de esa opción política, por lo que su política de seguridad se caracteriza por la convergencia con aquella de sus socios en la ASEAN.

\section{Relaciones del SEA con Japón}

El SEA es un área estratégica para Japón. Alrededor del $80 \%$ de sus importaciones de petróleo y un $70 \%$ de su comercio de bienes pasa por los estrechos de Málaca (Malasia-Singapur-Indonesia) y Lombok (Indonesia), que comunican el Océano Índico con el Pacífico ${ }^{24}$.

El Tratado de Paz de 1951 permitió reabrir las relaciones de Japón con la zona. El Primer Ministro Yoshida seguía viendo al SEA principalmente como fuente de materias primas, mientras en los temas de seguridad Tokio siguió los lineamientos de la política de "contención» de Estados Unidos, dirigida contra Moscú en el marco de la Guerra Fría.

La desconfianza y el resentimiento por las acciones japonesas en la II Gue-

24 Percival, B. (2006), "Japan-Southeast Asia Relations: Playing Catch-up with China», Comparative Connections: a Quarterly E-journal on East Asian Bilateral Relations, 8 (3), julio-septiembre, p. 162 <http:/www.csis.org/media/cesis/pubs/0603qiapan_seasia.pdf>. 
rra Mundial han sido un factor central que ha afectado una y otra vez las relaciones del SEA con Japón. En 1974 hubo manifestaciones de protesta en varios países (Tailandia, Malasia, Indonesia). En 1977, el Primer Ministro Takeo Fukuda anunció la doctrina que lleva su nombre como base de las relaciones niponas con el SEA. En ella se afirma que Japón rehúsa asumir el papel de potencia militar en el área; que procurará construir relaciones basadas en la confianza y el entendimiento; $y$ que se comportará como socio igualitario, cooperando con los países del área y con ASEAN en sus esfuerzos por fortalecer su solidaridad y su capacidad de adaptación a diversos cambios políticos y económicos. Desarrollando esta doctrina, Japón ha procurado contribuir a la estabilidad regional mediante el desarrollo económico impulsado por la inversión extranjera y la ayuda oficial para el desarrollo (ODA) ${ }^{25}$.

En 2003, el $50.6 \%$ de la ODA otorgada a la ASEAN era de origen nipón, con Indonesia como principal país receptor. Japón también se convirtió en principal socio comercial del SEA (salvo en Singapur y Filipinas). En el 2005, el comercio entre Japón y los países de la ASEAN se elevó a los $149.7 \mathrm{mil} \mathrm{mi-}$ llones de dólares, suma correspondiente al $18 \%$ del comercio total de la
ASEAN $^{26}$. Pese a que ha sido criticado, el rol de Japón en la recuperación del SEA tras la crisis financiera de 1997 ha sido importante. En 1999 creó un Fondo de Solidaridad Japón-ASEAN y en el 2000 el Fondo General de Intercambio Japón-ASEAN ${ }^{27}$.

En el 2003, la ASEAN y Japón acordaron un Plan de Acción en áreas económicas y financieras, políticas y de seguridad, y de cooperación cultural. Dentro de sus compromisos específicos se cuenta la donación por parte de Japón de 1.5 millones de dólares al proyecto de Desarrollo de la región del Mekong, en un plazo de tres años ${ }^{28}$. Igualmente, el Plan contempla la firma de Acuerdos de 'Partnership' Económico (EPA) bilaterales con los países del SEA. Hasta ahora, se han concluido negociaciones de EPA con Singapur y Malasia, se ha acordado el texto final del acuerdo con Tailandia y se encuentran en negociación con Filipinas, Indonesia y Brunei ${ }^{29}$.

El Primer Ministro Koizumi -quien considera a Fukuda como su mentor político-sostuvo la tesis de «actuar juntos y avanzar juntos" (2002), mientras que el Emperador Akihito ha asegurado que Japón es un país pacífico, y que no se repetirán los horrores de una guerra «muy desafortunada» (1991). Sin embargo, estos pronunciamientos no

2s Percival, B. (2006), p. 162.

26 Percival, B. (2006), p. 163.

27 Ministro de Relaciones Exteriores de Japón (MOFA), (2006), Japan-ASEAN relations: Ovcrview <http://www.mofa.go.jp/region/asia-paci/asean/relation/overview.htmls.

28 MOFA (2006).

29 Percival, B. (2006), p. 167. 
han bastado para eliminar el trasfondo de desconfianza que aún persiste. Una consecuencia de este clima de percepciones es que la reacción de los países de la ASEAN a las pretensiones japonesas de tener un rol político internacional más activo -especialmente a tener un asiento permanente en el Consejo de Seguridad de la ONU- no es de claro apoyo. Al parecer, solo Singapur apoya a Japón en sus aspiraciones. ${ }^{30}$

Irónicamente, la ASEAN ha promovido una mayor participación de Japón en las operaciones de paz de las Naciones Unidas en la zona y en operaciones contra amenazas no tradicionales tales como la lucha contra la piratería en el Mar del Sur de China ${ }^{31}$. Japón contribuyó con dinero y tropas de las Fuerzas de Autodefensa japonesas a las operaciones de paz en Camboya en 1992 y Timor Oriental en 2000 y $2002^{32}$. En 2004, Japón y la ASEAN aprobaron una Declaración conjunta sobre cooperación para combatir el terrorismo internacional; en 2005, el Primer Ministro Koizumi se comprometió a donar 130 millones de dólares para la lucha contra enfermedades infecciosas y en marzo de 2006 creó el Fondo de Integración Japón-ASEAN, que busca fortalecer los esfuerzos de la ASEAN para manejar problemas regionales tales como la gripe aviar y el terrorismo. Japón contribuyó 170 millones de dólares a dicho fondo ${ }^{33}$.

La relación entre los países de la ASEAN y Japón se mueve principalmente en los temas de inversión, comercio y ayuda financiera, por lo cual los países del SEA perciben a Japón como un 'gigante económico y un enano político' en la región ${ }^{34}$. Por ejemplo, Japón participa activamente en los foros y organismos multilaterales del este de Asia, como ASEAN+1, ASEAN+3, ARF, y la Cumbre del Este de Asia, en los cuales lidera los temas financieros, dejando a la ASEAN el liderazgo del proceso, situación que es bienvenida por los países del SEA ${ }^{35}$.

Es importante tener en cuenta que muchas de las actuaciones de Japón en el área están guiadas por una creciente competencia con China por tener influencia en la región ${ }^{36}$. Cabe recalcar que Japón accedió al Tratado de Amistad y Cooperación en el año 2004, luego de que China hiciera lo propio en el 2003. Igualmente, el Plan de Acción ASEAN-Japón, ya mencionado, fue propuesto luego de que China propusiera la firma de un TLC con la ASEAN. 


\section{CONClusiones}

La subregión del SEA se ha beneficiado con el fin de la Guerra Fría, en términos de potencial de cooperación y espacios de maniobra internacional. No obstante, una y otra vez afloran tensiones y problemas entre algunos de sus miembros (tema que no hemos tratado en este artículo). Por otra parte, la ASEAN, como entidad que agrupa a países medianos y pequeños, no puede ignorar la influencia de las potencias mayores, independientemente de las percepciones acerca de sus políticas (hegemónicas o no hegemónicas).

Por otra parte, a partir de septiembre de 2001, nuevos factores de inestabilidad amenazan a la subregión, particularmente Indonesia y otros países, en los cuales grupos terroristas han infiltrado los movimientos musulmanes pacíficos.

Las principales potencias que influyen en la región son Estados Unidos y China. Los países del SEA quieren evitar tener que "optar" entre ambas potencias. La potencia que pretenda que el SEA se incline hacia su campo de influencia probablemente perderá gravitación, porque el acto mismo de tratar de forzar una opción se considerará como prueba de sus designios hegemónicos. La clave de la fortaleza de la posición regional de China es (irónicamente) la credibilidad de su imagen de socio pacífico, cooperador y no excluyente. Por esta razón, la mejor política para China sería mantener la orientación de su política actual, aun en un contexto de rivalidad global.
En el marco de la normalidad que se observa en las relaciones entre China y el SEA, es dudoso que Estados Unidos pueda contener la influencia china en la región. Pero si China cambiara su política y ejerciera presión sobre los países del SEA para obtener su apoyo en un conflicto mayor, tal conducta sería mal vista por sus vecinos, y por ende China estaría "conteniêndose" a sí misma.

Por su parte, China también compite con Japón por mayor influencia en la región. El papel de este último se ha limitado a proveer recursos económicos y oportunidades comerciales, lo cual ha permitido que China lo haya desplazado gradualmente de la zona.

Concluiremos señalando que en el nuevo contexto político-económico global y regional los países de ASEAN se han convencido de que, para tener algún peso colectivo internacional deben modernizar sus instituciones. De esta manera, han decidido adoptar una Carta de la ASEAN, en la cual se codifiquen las reglas, normas y valores que lo rigen, y se ratifiquen los tratados, acuerdos y otros instrumentos aprobados en su seno. En otras palabras, la Carta proveerá de marco institucional a un organismo que desde su concepción ha evitado los formalismos y rigideces de los esquemas de integración occidentales. Está por verse cuáles serán las consecuencias de este nuevo camino por la ASEAN y sus implicaciones para la región y su vinculación al este de Asia. 